\title{
Structures of riboswitch RNA reaction states by mix-and-inject XFEL serial crystallography
}

J.R. Stagno ${ }^{1}$, Y. Liu ${ }^{1}$, Y.R. Bhandari ${ }^{1}$, C.E. Conrad ${ }^{2,3}$, S. Panja ${ }^{4}$, M. Swain ${ }^{1}$, L. Fan ${ }^{5}$, G. Nelson ${ }^{6}$, C. Li ${ }^{6}$, D.R.

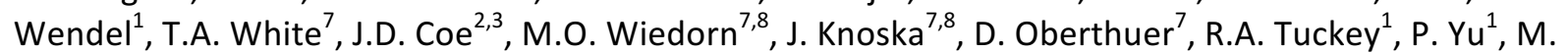
Dyba $^{1}$, S.G. Tarasov ${ }^{1}$, U. Weierstall ${ }^{3,6}$, T.D. Grant ${ }^{9}$, C.D. Schwieters ${ }^{10}$, J. Zhang ${ }^{11}$, A.R. Ferré-D'Amaré ${ }^{12}$, P. Fromme $^{2,3}$, D.E. Draper ${ }^{13}$, M. Liang $^{14}$, M.S. Hunter ${ }^{14}$, S. Boutet ${ }^{14}$, K. Tan $^{15}$, X. Zuo ${ }^{16}$, X. Ji $^{17}$, A. Barty ${ }^{7}$, N.A. Zatsepin $^{3,6}$, H. N. Chapman ${ }^{7,8}$, J.C.H. Spence ${ }^{3,6}$, S. A. Woodson ${ }^{4}$ \& Y.-X. Wang ${ }^{1 *}$

${ }^{1}$ Structural Biophysics Laboratory, Center for Cancer Research, National Cancer Institute, Frederick, MD 21702, USA; ${ }^{2}$ Department of Biochemistry, Arizona State University, Tempe, AZ 85287, USA; ${ }^{3}$ Center for Applied Structural Discovery, The Biodesign Institute, Arizona State University, Tempe, AZ, 85287, USA; ${ }^{4}$ Department of Biophysics, Johns Hopkins University, Baltimore, MD 21218; ${ }^{5}$ Small Angle X-ray Scattering Core Facility, Center for Cancer Research, National Cancer Institute, Frederick, MD 21702 , USA; ${ }^{6}$ Department of Physics, Arizona State University, Tempe, AZ 85287, USA; ${ }^{7}$ Center for Free-Electron Laser Science, Deutsches Elektronen-Synchrotron DESY, Notkestraße 85, 22607 Hamburg, Germany; ${ }^{8}$ Hamburg Centre of Ultrafast Imaging and Department of Physics, University of Hamburg, Luruper Chaussee 149, 22607 Hamburg, Germany; ${ }^{9}$ Hauptmann-Woodward Medical Research Institute, Buffalo, NY 14203, USA; ${ }^{10}$ Center for Information Technology, National Institutes of Health, Bethesda, MD 20892-5624, USA; ${ }^{11}$ Laboratory of Molecular Biology, National Institute of Diabetes and Digestive and Kidney Diseases, ${ }^{12}$ Laboratory of RNA Biophysics and Cellular Physiology, National Heart Lung and Blood Institute, National Institutes of Health, Bethesda, MD 20892, USA. ${ }^{13}$ Department of Chemistry, Johns Hopkins University, Baltimore, Maryland 21218, USA; ${ }^{14}$ Linac Coherent Light Source, SLAC National Accelerator Laboratory, Menlo Park, California, USA; ${ }^{15}$ Structural Biology Center, Biosciences Division, ${ }^{16}$ X-ray Science Division, Advanced Photon Source, Argonne National Laboratory, Argonne, IL 60439, USA; ${ }^{17}$ Macromolecular Crystallography Laboratory, Center for Cancer Research, National Cancer Institute, Frederick, MD 21702, USA.

Riboswitches is RNA structural elements generally located in the 5 ' untranslated region (3'UTR) of mRNA. In the genetic regulation, ligand binding to the aptamer domain of a riboswitch triggers a signal to the downstream expression platform. A complete understanding of the structural basis for this mechanism requires the ability to study structural changes over time. We apply femtosecond X-ray free electron laser (XFEL) pulses to obtain structural measurements from crystals so small that diffusion of a ligand can be timed to initiate a reaction prior to diffraction. We demonstrate this approach by determining four structures of the adenine riboswitch aptamer domain during the course of a reaction involving two apo, one ligand-bound intermediate, and the final bound states. These structures support a reaction mechanism model with at least four states and illustrate the structural basis for signal transmission. The two apo conformers differ significantly in the three-way junction and the P1 switch helix relative to the ligand-bound conformation. Our time-resolved crystallographic measurements with a 10second delay captured the structure of an intermediate with changes in the binding pocket that accommodate the ligand. With a $>10$-minute delay, the RNA molecules were fully converted to the bound state, in which the substantial conformational changes resulted in conversion of the space group. Such drastic changes in crystallo highlight the important opportunities that micro/nanocrystals may offer in these and similar time-resolved diffraction studies. These results all together demonstrate the potential of "mix-and-inject" time-resolved serial crystallography to study biochemically important interactions between biomacromolecules and ligands, including those involving large conformational changes. 